\title{
Surgical management of intracranial Hodgkin's disease
}

\author{
T. F. BUCKLEY AND F. WARWICK
}

From the Departments of Neurological Surgery and Radiology, Radcliffe Infirmary, Oxford

The value of surgical intervention in compressive lesions of the spinal cord due to Hodgkin's disease is well recognized, but the role of surgery in the intracranial manifestations of the disease is not as well defined, probably because they are so rare. A search through the world literature has yielded only 23 cases of space-occupying lesions complicating Hodgkin's disease. Only seven of these patients were operated upon. The following case is of interest in that extirpation of an intracranial Hodgkin's granuloma was performed. It was the only lesion of this type seen at this hospital during the past 30 years, although several examples of spinal cord compression in Hodgkin's disease have been encountered.

\section{CASE REPORT}

H. E. (Hosp. No. 413726), a builder by trade, first attended hospital in June 1963 when he was 36 years old, with a six months' history of swelling on the right side of his neck, recently associated with generalized pruritis. The diagnosis of Hodgkin's disease was confirmed by biopsy of lymph nodes in the right supraclavicular fossa, and a course of deep $x$-ray treatment to the right side of the neck and the mediastinum followed. A further course of deep $x$-ray treatment to the right side of the neck was given six months later (December 1963-January 1964). From May to June 1964 a course of chemotherapy was given: $700 \mathrm{mg}$ mannomustine hydrochloride (Degranol) intravenously. Between July 1964 and July 1966 he received successive courses of irradiation to the right submaxillary region, left side of the neck, abdomen, right iliac fossa, left iliac fossa, and right axilla.

$\mathrm{He}$ remained reasonably well during this time and continued at work intermittently till November 1966, when a nocturnal grand mal epileptic fit occurred. A further fit followed one week later, after which the left upper limb seemed weak. He was admitted to hospital again in January 1967, but examination revealed no evidence of recrudescence of Hodgkin's disease, although the ESR was $67 \mathrm{~mm} / 1 \mathrm{hr}$. Neurological examination was also normal, but an electroencephalogram suggested a possible space-occupying lesion in the right frontal area. Lumbar puncture yielded a clear CSF at a pressure of $120 \mathrm{~mm}$ of water and the fluid contained $43 \mathrm{mg}$ of protein/ $100 \mathrm{ml}$. with a normal cell count. A Jacksonian fit affecting the left side of the face and the left limbs occurred at the end of January 1967, and a progressive left hemiparesis ensued affecting the lower limb more than the upper limb or face. His mental processes became slow at this time, but there were no symptoms suggestive of raised intracranial pressure. He was transferred to the Department of Neurological Surgery at the Radcliffe Infirmary on 21 February 1967. On admission, he was found to be conscious and orientated, but mentally slow and apathetic. There was no evidence of raised intracranial pressure and he denied any headache. He had a pronounced left hemiparesis affecting the lower limb more than the face or upper limb, with corresponding alteration in the tendon reflexes, and slight sensory impairment on the left side of the body. Plain radiographs of the skull revealed slights erosion of the floor of the pituitary fossa suggestive of $N$ raised intracranial pressure; a dilated right middle meningeal channel raised the possibility of a parasagittag meningioma in the fronto-parietal region. A right carotiof angiogram was done (Fig. 1) and this clearly outlined ait avascular mass in the fronto-parietal parasagittal regiono apparently extracerebral, with some extension across the midline to the left side. The superior sagittal sinus appeared to be implicated as it did not fill anteriorly and the overlying bone also seemed to be involved.

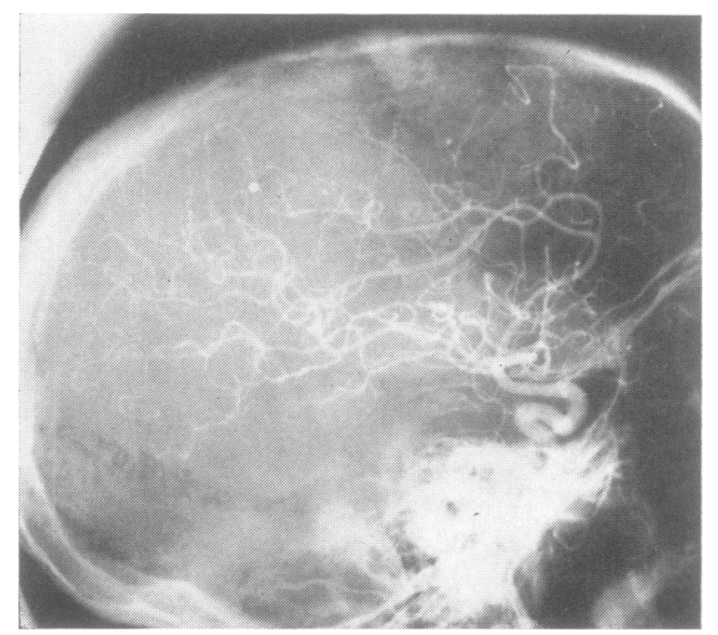

FIG. 1. Lateral carotid arteriogram outlining avascular frontal parasagittal mass. 
Through a right lateral flap extending to the midline, this area was explored on 23 February 1967. The dura was adherent to the bone along the midline and when the bone flap was reflected a large mass of tumour $(4 \mathrm{~cm}$ in diameter) adherent to the bone was elevated with it. The torn surface of the underlying tumour was quite avascular and yellowish-grey in colour. It was a firm fibrous mass, adhering to the falx and superior sagittal sinus as well as to the parasagittal dura. It measured $9 \mathrm{~cm}$ from front to back, $5 \mathrm{~cm}$ from side to side, and extended vertically along the side of the falx for $5 \mathrm{~cm}$. The main mass was dissected free of the adjoining cortex and it came easily with minimal decortication along the supero-medial margin of the hemisphere. Its medial margin was easily dissected off the falx, but the superior sagittal sinus appeared to be involved by the tumour. The sinus was preserved, the tumour being incised flush with the sinus edge, as it was deemed safer to deal with any residual tumour by subsequent irradiation. The mass attached to the bone flap was removed together with its adherent dura. It was noted that there was dense adhesion to the bone with little spicules of endostosis. The pathological area of bone was also excised. The defect in the parasagittal dura measured $10 \mathrm{~cm}$ by $8 \mathrm{~cm}$ and was repaired with fascia lata. To the naked eye, the appearances at operation were those of a rather 'wild' parasagittal meningioma.

During the succeeding days, power rapidly returned in the left lower limb, the weakness of the upper limb receding rather more slowly. Phenytoin, $100 \mathrm{mg}$ t.d.s., was administered, but there were no further fits. $\mathrm{He}$ was transferred back to his referring hospital on 1 March 1967 , where a course of tele-cobalt treatment to the head was commenced on the following day, as well as further deep $x$-ray treatment to the abdomen and left groin. His condition improved considerably and when seen as an outpatient at the radiotherapy unit on 2 May 1967, he was feeling much better and walking well with no evident weakness of the left side. Three weeks later (25 May 1967) he was re-admitted for thoracentesis: a right pleural effusion of $1,600 \mathrm{ml}$. turbid yellow fluid was aspirated and $15 \mathrm{mg}$ nitrogen instilled. A blood transfusion was then given, but he continued to deteriorate, had two further generalized fits early in June, and died at home on 9 June 1967-3 - $\frac{1}{2}$ months after operation. A necropsy was not carried out.

The histology report on the excised tumour, which weighed more than $80 \mathrm{~g}$, confirmed that the tumour was infiltrating the dura as well as the overlying skull (Fig. 2). The histological appearances were pleomorphic, with a predominance of large irregular pale-staining cells with processes. Among these cells were binucleated and multinucleated giant cells, and the picture was completed by a variety of other cell-types such as lymphocytes, plasma cells, and eosinophils. The tumour was quite cellular and from one or two mitotic figures were found per high power field (Fig. 3). The appearances were consistent with a Hodgkin's granuloma.

\section{DISCUSSION}

The incidence of neurologically significant complications in Hodgkin's disease is variously reported:
Diamond (1957), cited $13 \%$ and Winkleman and Moore (1941) $62.5 \%$, while a review of the last 100 cases of the disease seen at the Radcliffe Infirmary showed a $30 \%$ incidence of significant involvement of the nervous system. Cerebral manifestations are considerably less frequent, ranging from $1.6 \%$ (Diamond, 1957) to 10\% (Hutchinson, Leonard, Maudsley, and Yates, 1958), or $12 \%$ (Radcliffe Infirmary series). These cerebral manifestations can be due to widely differing processes, many of which may simulate a space-occupying lesion. The pathological processes may be listed as follows:

A. DEMONSTRABLE NEOPLASTIC INVOLVEMENT (1) Of soft tissues of head and neck - tumours may involve the cranial nerves issuing through skull foramina; the fifth, sixth, seventh, and twelfth cranial nerves are those most often affected (Diamond, 1957).

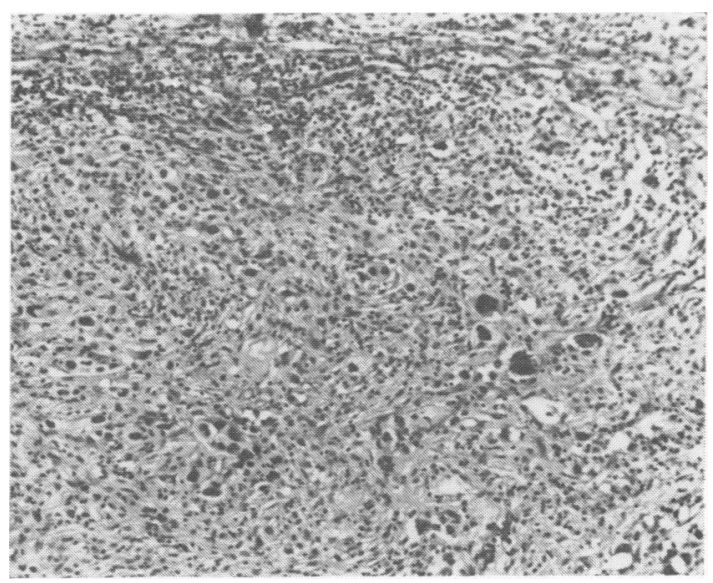

FIG. 2. Histological appearances of excised tumour $(\times 77)$.

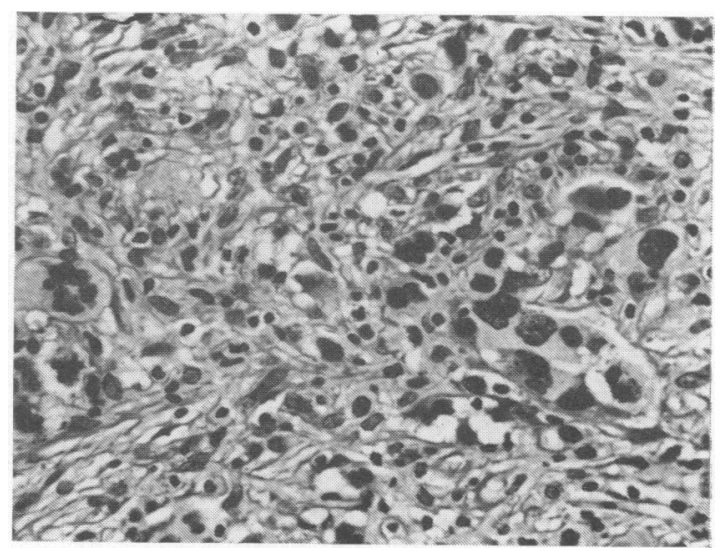

FIG. 3. High-power view of same $(\times 240)$. 
(2) Of bone-with secondary compression of cranial nerves or brain.

(3) Of dura-either diffuse infiltration or plaque or mass formation.

(4) Of cranial nerve roots-discrete infiltration by Hodgkin's tissue.

(5) Of brain-(a) diffuse cerebral lesions such as meningoencephalitis or multiple tiny foci of Hodgkin's tissue; (b) discrete intracranial masses.

B. DEMONSTRABLE VASCULAR Lesions For example, cerebral haemorrhage, 'cerebral purpura', or chronic subdural haematoma. There may be associated blood dyscrasias such as thrombocytopaenia.

C. DEMONSTRABLE INFECTIVE Lesions Often due to organisms of normally low infectivity-for example, Torula meningitis, herpes zoster meningoencephalitis, or tuberculous cerebral abscess.

D. OTHER DEMONSTRABLE LESIONS For example, focal demyelination (leucoencephalopathy).

E. CONCOMITANT LESIONS UNRELATED TO HODGKIN'S DISEASE For example, intracranial meningioma (Lascelles and Burston, 1962).

F. NO DEMONSTRABLE LESION AT NECROPSY. It has long been recognized that cerebral syndromes may occur in Hodgkin's disease without any abnormality being detected at necropsy. Hence Sparling, Adams, and Parker (1947) emphasized that it is mandatory to obtain histological confirmation of any suspected space-occupying lesion which may occur during the course of the disease, either at operation or necropsy, before accepting the lesion as being truly spaceoccupying. This was reiterated by John and Nabarro (1955). Using this criterion, we have been able to find only 22 well-documented cases of intracranial space-occupying Hodgkin's lesions in a review of the world literature.

These tumours can be placed in four groups: (1) extradural tumours with secondary involvement of dura and brain, (2) dural tumours, (3) tumours involving dura and brain, (4) intracerebral tumours.

Hodgkin's disease in the cranial bones with subsequent formation of a mass within the cranium appears to be an exceedingly rare occurrence, and only one case has been reported (Fein and Newill, 1954). Dural infiltration from extradural deposits is not uncommon and these patients often develop cranial nerve palsies or diabetes insipidus during the course of their disease (Lascelles and Burston, 1962). One case of an asymptomatic dural granuloma was discovered at necropsy by Martin and Courville (1936). Nine cases of purely intracerebral mass lesions in Hodgkin's disease have been reported and 11 cases of masses involving the dura and brain; of these 20 cases, the cerebral lesion was the initial manifestation of Hodgkin's disease in at least seven cases. The case reported here is a further example of a Hodgkin's tumour involving the dura and brain, secondary to generalized Hodgkin's disease.

The histological grouping in Hodgkin's disease of paragranuloma, granuloma, and sarcoma is now generally accepted (Ljungdahl, Strang, and Tovi, 1965). Sparling et al. (1947) stated that there was no reported case of involvement of the central nervous system by Hodgkin's paragranuloma. Hutchinson et al. (1958) confirmed this in their analysis of 168 cases of Hodgkin's disease; eight paragranulomata were included, none of which involved the central nervous system. As far as we have been able to ascertain, a case of paragranuloma involving the CNS has yet to be reported. The intracranial tumours occurring in Hodgkin's disease appear to be almost equally divided between granulomata and sarcomata in those cases which have been distinguished histologically.

The management of these lesions may require considerable clinical judgment if the neurologica 0 complications present late in the course of the $\vec{N}$ disease. In those cases where it is possible to do so용을 an attempt should be made to define the exact nature $\overrightarrow{ }$ of the lesion. The cases which present with primary cerebral involvement should be investigated fully and $\overparen{\AA}$ a histological diagnosis obtained. This has usuallyo entailed a craniotomy or burrhole biopsy. Three cases have been reported of surgical extirpation of primary intracranial Hodgkin's tumour.

The first recorded case (Sparling and Adams, 1946) had a three-month history of progressive confusion, dysphasia, fits, and right hemiplegia; the patient died of meningitis five days after excision of a mass of Hodgkin's sarcoma from the left frontal lobe. The second case was that of a 45-year-old man with a nine-month history of mental change and recent raised intracranial pressure, from whose right temporal lobe a Hodgkin's granuloma was excised, and who, after post-operative irradiation (1500 rads), remained well three years later without evidence of Hodgkin's disease elsewhere (Schricker and Smith, 1955). The third patient was a 23-year-old woman with a two-month history of headache, vomiting, and papilloedema, who recovered completely after removal of a left frontal mass attached to the dura and invading the brain, but developed enlarged glands in the neck and mediastinum seven months later (Boixadós, Córdoba, and Roda, 1963).

The role of surgery in the management of intracranial tumours complicating previously diagnosed Hodgkin's disease is not fully resolved. Although 
Hunt, Bouroncle, and Meagher (1959) favour initial radiation with surgical intervention only if the patient fails to respond or becomes worse, there is little doubt that histological confirmation should be obtained before instituting any treatment, and indeed, a case can be made for surgical extirpation in those patients whose systemic manifestations appear well controlled and who do not have associated blood dyscrasias. Ljungdahl et al. (1965) described a 29year-old woman with known Hodgkin's disease for four years who was treated with thiotepa when she developed headache and paraesthesiae in her left upper limb. One year later her symptoms recurred with weakness and sensory loss in the left upper limb. An intracerebral granuloma, the size of a hen's egg, was removed from the right parietal lobe followed by local irradiation. She remained well three years later. John and Nabarro (1955) cited a 25-year-old man with Hodgkin's disease of four years' duration, which had been treated with local radiation and systemic nitrogen mustard. When headache, misty vision, and papilloedema supervened, systemicnitrogen mustard produced transient improvement, but he was readmitted three months later and a mass of Hodgkin's tissue was partially excised from the right middle fossa, where it was attached to the dura and invading the posterior part of the right cerebral hemisphere. The patient recovered, and raised intracranial pressure did not recur before his death 14 months later, although he did have an episode of hiccoughing which responded to a two-day course of radiation directed to his medulla. Vogel and Richland (1955) recorded the case of a 34-year-old woman who had a Hodgkin's granuloma excised from her thoracic spine five years before focal fits led to excision of a left parietal granuloma attached to the dura and compressing the underlying brain. She was recovering well two months later without evidence of generalized disease. From these few reports, it would appear that surgical extirpation of Hodgkin's tumours within the cranium may be of benefit in some cases. Such are those in which there is evidence of increased intracranial pressure or of progressive neurological deficit in patients whose disease is otherwise well controlled. As total removal is usually impossible, post-operative radiation treatment is almost always necessary.

The effect of radiation alone on an intracranial Hodgkin's mass has been described by Hunt et al. (1959): their patient, a 20-year-old man with known Hodgkin's disease for four years, developed a profound left hemisphere syndrome with raised intracranial pressure, but surgery was precluded by severe thrombocytopaenia. A course of deep radiation to the left temperoparietal region caused an initial deterioration in the level of consciousness, but the patient subsequently showed marked neurological improvement, although dying of pulmonary infiltration two months later. At necropsy there was a scarred cystic area in the left temporo-parietal region with persisting Hodgkin's tissue in its wall.

\section{SUMMARY}

A case of intracranial Hodgkin's granuloma complicating generalized Hodgkin's disease in a 40-year-old man is reported. The causes of cerebral syndromes in Hodgkin's disease are reviewed, as are the various mass lesions that affect the brain in this disease. It is emphasized that histological verification is necessary before accepting a mass lesion as being a Hodgkin's tumour. Some of these tumours - whether a primary manifestation or secondary to pre-existing Hodgkin's disease-are amenable to surgical treatment. Radiation is a valuable adjunct to surgery or may be used alone when the nature of the lesion has been verified, particularly if surgery is contraindicated.

We are grateful to Mr. J. Pennybacker for his permission to publish this case and for his help in preparing this paper; also to Dr. P. W. E. Sheldon for the $x$-ray studies, to Dr. J. Trevor Hughes for the photomicrographs, and to Dr. B. Jolles for details for the radiation treatment.

\section{REFERENCES}

Boixadós, J. R., Córdoba, A., and Roda, E. (1963). Consideraciones sobre un caso de granuloma de Hodgkin de manifestacion primeria meningocerebral. Rev. clin. esp., 90, 46-50.

Diamond, H. D. (1957). Hodgkin's disease: neurologic sequelae. Missouri Med., 54, 945-956.

Fein, S. B., and Newill, V. A. (1954). Cerebral Hodgkin's disease. Case report of Hodgkin's granuloma with cerebral invasion. Amer. J. Med., 17, 291-294.

Hunt, W. E., Bouroncle, B. A., and Meagher, J. N. (1959). Neurologic complications of leukemias and lymphomas. J. Neurosurg., 16, 135-151.

Hutchinson, E. C., Leonard, B. J., Maudsley, C., and Yates, P. O. (1958). Neurological complications of reticuloses. Brain, 81, 75-92.

John, H. T., and Nabarro, J. D. N. (1955). Intracranial manifestations of malignant lymphoma. Brit. J. Cancer, 9, 386-400.

Lascelles, R. G., and Burston, J. (1962). Hodgkin's disease. Disease presenting with symptoms of cranial nerve involvement. Arch. Neurol. (Chic.), 7, 359-364.

Ljungdahl, I., Strang, R. R., and Tovi, D. (1965). Intracerebral Hodgkin's granuloma. Report of a case and review of the literature. Neurochirurg., 8, 113-118.

Martin, H. E., and Courville, C. B. (1936). Hodgkin's disease with involvement of the cranial dura mater. Bull. Los Angeles Neurol. Soc., 1, 145-148.

Schricker, J. L., and Smith, D. E. (1955). Primary intracerebral Hodgkin's disease. Cancer, 8, 629-633.

Sparling, H. J., and Adams, R. D. (1946). Primary Hodgkin's sarcoma of the brain. Arch. Path., 42, 338-344.

,-- , and Parker, F. (1947). Involvement of the nervous system by malignant lymphoma. Medicine (Baltimore), 26, 285-332.

Vogel, P. J., and Richland, K. J. (1955). Involvement of the central nervous system by Hodgkin's disease. Case report with five year interval between primary spinal and cerebral lesions. Bull. Los Angeles neurol. Soc., 20, 83-86.

Winkleman, N. W., and Moore, M. T. (1941). Lymphogranulomatosis (Hodgkin's disease) of the nervous system. Arch. Neurol. Psychiat. (Chic.), 45, 304-318. 